\title{
Imaging features of breast cancer molecular subtypes: state of the art
}

\author{
Nariya Cho ${ }^{1,2,3}$ \\ 'Department of Radiology, Seoul National University Hospital, Seoul; \\ 2Department of Radiology, Seoul National University College of Medicine, Seoul; \\ ${ }^{3}$ Institute of Radiation Medicine, Seoul National University Medical Research Center, Seoul, Korea
}

Characterization of breast cancer molecular subtypes has been the standard of care for breast cancer management. We aimed to provide a review of imaging features of breast cancer molecular subtypes for the field of precision medicine. We also provide an update on the recent progress in precision medicine for breast cancer, implications for imaging, and recent observations in longitudinal functional imaging with radiomics.

Key Words: Breast neoplasms; Magnetic resonance imaging; Gene expression profiles

Received: August 9, 2020 Revised: September 3, 2020 Accepted: September 6, 2020

Corresponding Author: Nariya Cho, MD, PhD, Department of Radiology, Seoul National University Hospital, 101 Daehak-ro, Jongno-gu, Seoul 03080, Korea Tel: +82-2-2072-1862, Fax: +82-2-743-6385, E-mail: river7774@gmail.com

Gene expression profiling has revealed that there are four major breast cancer subtypes: luminal A, luminal B, human epidermal growth factor receptor 2 (HER2)-enriched, and basal-like tumors [1-3]. Each subtype has varied prognoses, risk of progression, response to treatment, and survival outcomes. In general, basal-like tumors have the worst prognosis, while luminal A tumors have the best prognosis. However, as full genomic analysis is costly and time consuming in clinical practice, the St. Gallen International Expert Consensus panel has suggested surrogate subtypes based on semiquantitative immunohistochemistry (IHC) scoring of estrogen receptor (ER), progesterone receptor (PR), and in situ hybridization tests for HER2 overexpression as follows: luminal A (ER and/or PR positive, HER2 negative), luminal B (ER and/or PR positive and HER2 positive or Ki67 $\geq 14 \%$ ), HER2-enriched (HER2 amplified, ER and PR negative), and triple-negative breast cancer (TNBC; ER, PR, and HER2 negative) [4-6] (Table 1). Type determination by percutaneous imageguided biopsy is the first step in managing systemic therapy strategy for breast cancer, because traditional prognostic factors including tumor size, histologic grade, and lymph node status do not fully reflect the heterogeneity of breast cancer, and treatment guidelines are no longer based solely on anatomic stage. The biological diversity of tumors requires the continual refinement of treatment algorithms, which are more and more person- alized in the recent St. Gallen Consensus Guidelines [6]. The escalating strategy includes longer duration of anti-estrogen therapy, ovarian function suppression, dual blockade with antiHER2 therapy, and residual tumor treatment following neoadjuvant chemotherapy [6]. The de-escalating strategy includes omission of adjuvant chemotherapy, shortening of radiation therapy, and avoidance of axillary dissection [6]. However, percutaneous biopsy sampling does not represent the topographic heterogeneity of a whole tumor. Moreover, as breast cancer continuously evolves following systemic therapy, spatio-longitudinal monitoring of a whole tumor using imaging modalities during systemic therapy is crucial.

The earliest imaging studies have reported that the triple-negative subtype has non-calcified and circumscribed margins, the luminal subtype mass is irregular with spiculated margins, and the HER2-positive subtype mass has pleomorphic calcifications [7]. While repeated measurements of voxel-based signal intensity of whole tumor is feasible, with breast magnetic resonance imaging (MRI) there are several studies that link imaging phenotypes using radiomics analysis with breast cancer molecular subtypes. In this article, we aim to help readers to stay up-to-date and play a role as key members of a multidisciplinary team for breast cancer treatment. 


\section{RADIOMICS IN BREAST CANCER}

Radiomics is a technique to extract and select the quantitative features of radiologic images, to create a high-dimensional data set, and to draw hypotheses, which will lead to better clinical decisions [8]. In breast radiomics analysis, determination of benign or malignant lesion, correlation with prognostic factors, prediction of the response to systemic chemotherapy or lymph node metastasis have been studied. Radiomics features are mathematically defined and classified into morphology, histogram, texture, or transformed features [8]. Morphology features are compactness, roundness, or convexity. Histogram features are median, entropy, uniformity, skewness, or kurtosis, in which spatial information is not included. Conversely, texture features include the spatial information. Gray-level co-occurrence matrix (GLCM)-based features are the most commonly used method for textural analysis [8]. The relationship between voxels and their neighborhoods are characterized in GLCM analysis. Entropy, contrast, and homogeneity, which reflect the uniformity or heterogeneity of the voxel signal intensities, are the main parameters of GLCM models. Transform-based features such as Laplacian of Gaussian and wavelet are commonly used; these transform the original image, creating a new image from which the features can be quantified.

Application of radiomics in distinguishing molecular subtypes is one of the most intensely studied areas (Table 2) [9-20]. Leithner et al. [9] reported accuracies of $81 \%-89 \%$ in distinguishing luminal A from luminal B, luminal B from triplenegative, luminal B from all others, and HER2-enriched from all others. In their study, the region of interest of a tumor was drawn, and gray-level normalization was performed to minimize the effect of contrast variations. Then, first-order histogram, GLCM, and transform-based features were calculated. Feature selection to reduce the dimensionality of texture features, to select the minimal numbers of features explaining the phenomenon, were performed. K-nearest neighbor classification with leave-one-out cross validation was performed for classification [9]. More recent studies using radiomic analysis reported an area under the receiver operating characteristic curve (AUC) of 0.844 in the prediction of disease-free survival of TNBC [10] and an AUC of 0.890 in the differentiation of HER2-positive tumors vs. -negative tumors [11]. The radiomics approach is based on the premise that microstructural variations between molecular subtypes would cause various gray-level textures on contrast enhanced MRI. The radiomics approach has the potential to provide prognostic information of spatio-longitudinal

Table 1. Treatment-oriented classification of subgroups of breast cancer from St. Gallen consensus guidelines

\begin{tabular}{|c|c|c|}
\hline Clinical grouping & Notes & Type of therapy \\
\hline Triple-negative & Negative ER, PR, and HER2 & $\begin{array}{l}\text { Cytotoxic chemotherapy including anthracycline and taxane } \\
\text { Consideration of cabecitabine for residual tumor after } \\
\text { neoadjuvant chemotherapy }\end{array}$ \\
\hline ER negative and HER2 positive & $\begin{array}{l}\text { American Society of Clinical Oncology (ASCO)/College of } \\
\text { American Pathologists (CAP) guideline } 2018^{a}\end{array}$ & $\begin{array}{l}\text { Stage 1: paclitaxel+trastuzumab as adjuvant therapy } \\
\text { Stage } 2 \text { or } 3 \text { : Neoadjuvant anthracycline, alkylator-, and } \\
\text { taxane-based chemotherapy + trastuzumab- and } \\
\text { pertuzumab-based treatment (dual anti-HER2 therapy) } \\
\text { Trastuzumab ematansine therapy for residual tumor after } \\
\text { neoadjuvant chemotherapy in adjuvant setting }\end{array}$ \\
\hline ER positive and HER2 positive & ASCO/CAP guidelines 2018a & $\begin{array}{l}\text { As above +endocrine therapy appropriate to menopausal } \\
\text { status }\end{array}$ \\
\hline
\end{tabular}

\footnotetext{
ER positive and HER2 negative

Luminal A-like (high receptor,

ER and/or PR-positive $\geq 1 \%{ }^{\text {b }}$

low proliferation, low grade)

Multi-parameter molecular marker 'good' if availablec High ER/PR and clearly low Ki-67 or grade

Intermediate

Multi-parameter molecular marker 'intermediate' if available Endocrine therapy+adjuvant chemotherapy

Uncertainty persists about degree of risk and

responsiveness to endocrine and cytotoxic therapies

Luminal B-like (low receptor,

Multi-parameter molecular marker 'bad' if available. Lower

high proliferation, high grade) ER/PR with clearly high Ki-67, high histological grade 3

Endocrine therapy alone according to menopausal status

Endocrine therapy + adjuvant chemotherapy

Modified from Coates AS et al. Ann Oncol 2015;26:1533-46 [4] and Burstein HJ et al. Ann Oncol 2019;30:1542-57 [6], according to the Creative Commons license.

ER, estrogen receptor; PR, progesterone receptor; HER2, human epidermal growth factor receptor 2; TNBC, triple-negative breast cancer.

${ }^{a}$ An immunohistochemistry of c-erbB-2 staining $3+$ score was defined as HER2-positive, while a 0 or $1+$ score was negative. For tumors with $2+$ score, HER2 gene copies to the centromeric region of chromosome 17 ratios $<2.0$ by fluorescence in situ hybridization must be interpreted as negative due to the lack of evidence for any benefit from HER2 targeted therapy; blf ER values fall between $1 \%$ and $9 \%$, the term equivocal should not be used, suggesting response to endocrine therapy even in low ER (1\%-9\%). Low HR expression is associated with higher Ki-67, higher grade, and loss PR positivity, as well as higher recurrence score and higher chemo-sensitivity. Chemotherapy should be given following guidelines for TNBC. Endocrine therapy should be recommended despite the likely extremely small benefit; ' No role for gene testing in clinical pathologic low risk cases (pT1a, pT1b, G1, ER high, pNO).
} 

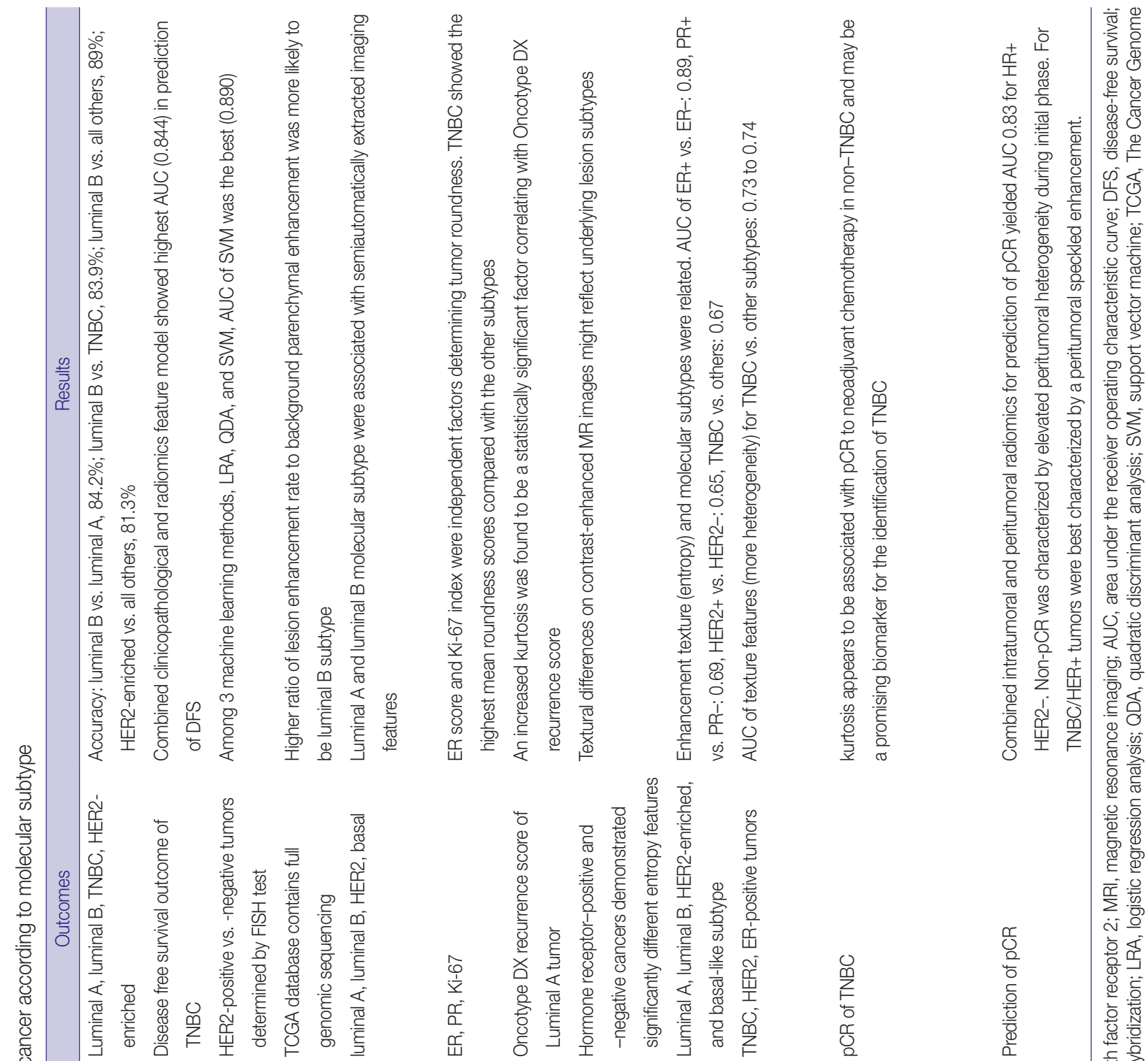

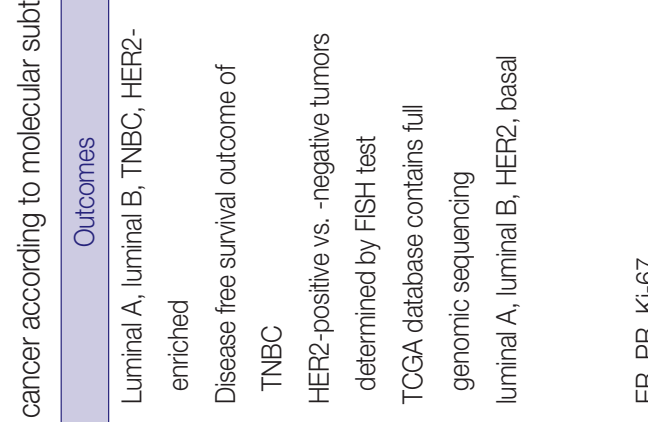

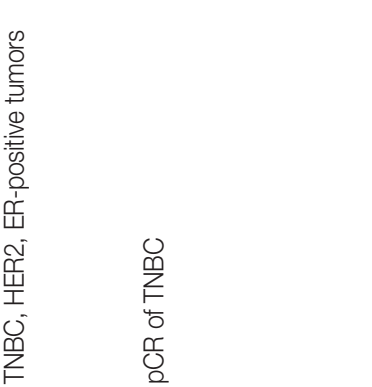

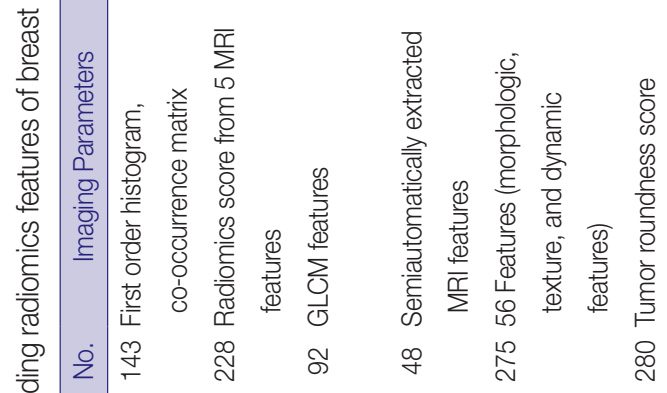

产产

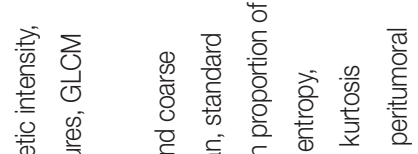

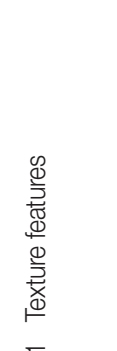

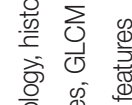

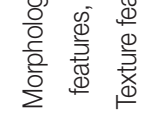

๑ స స

क

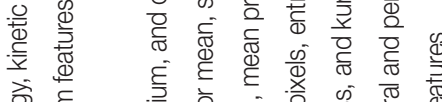

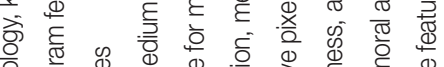

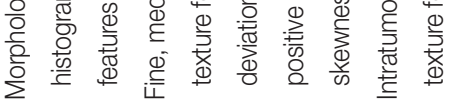


biology changes of the peritumoral parenchyma as well as of the tumor itself. Moreover, deep learning methods have increasingly been applied in recent radiomics studies $[8,11]$. The deep learning approach, which is data driven and capable of learning relevant features from the data themselves, has shown superior performance in various tasks in radiology $[8,11]$. In the near future, radiomics parameters based on a deep learning algorithm would be useful surrogate markers for precision medicine in breast cancer treatment.

\section{LUMINAL SUBTYPE}

About $70 \%$ of breast cancers are ER positive and show a more favorable prognosis than ER-negative cancers. Within ER-positive/HER2-negative breast cancer, 90\%-95\% are luminal A or B subtypes [3]. The luminal B subtype shows higher proliferation gene expression [2] and worse recurrence-free survival outcomes compared with the luminal A subtype, although the luminal B subtype shows higher pathological complete response
(pCR) rate following neoadjuvant chemotherapy [3]. Thus, differentiation between luminal A and B tumors is important for deciding the duration of endocrine therapy or to predict resistance to endocrine therapy [3]. There is a 30 to $44 \%$ discordance rate between the gene expression profiling and surrogate IHC classifications [3,21]. Within ER-positive/HER2-negative breast cancers, $5 \%-10 \%$ of tumors are non-luminal subtypes (HER2 enriched and basal-like tumors) by gene expression profiling [3]. Non-luminal (ER positive/HER2 negative) breast cancers show worse outcomes compared with the luminal A subtype when they were treated with 5 years of adjuvant tamoxifen-only [22]. One study reported that $80 \%$ of tumors showing low expression ER positive (1\%-9\%) were non-luminal subtypes [23].

For patients with ER-positive tumors, prognostic signatures including 70-gene MammaPrint microarray assay (Agendia, Amsterdam, The Netherlands), the 50-gene PAM50 assay (Prosigna, Nanostring Technologies, Seattle, WA, USA), and the 21-gene Oncotype DX assay (Genomic Health, Redwood City, CA, USA) are commercially available [24]. These signatures al-
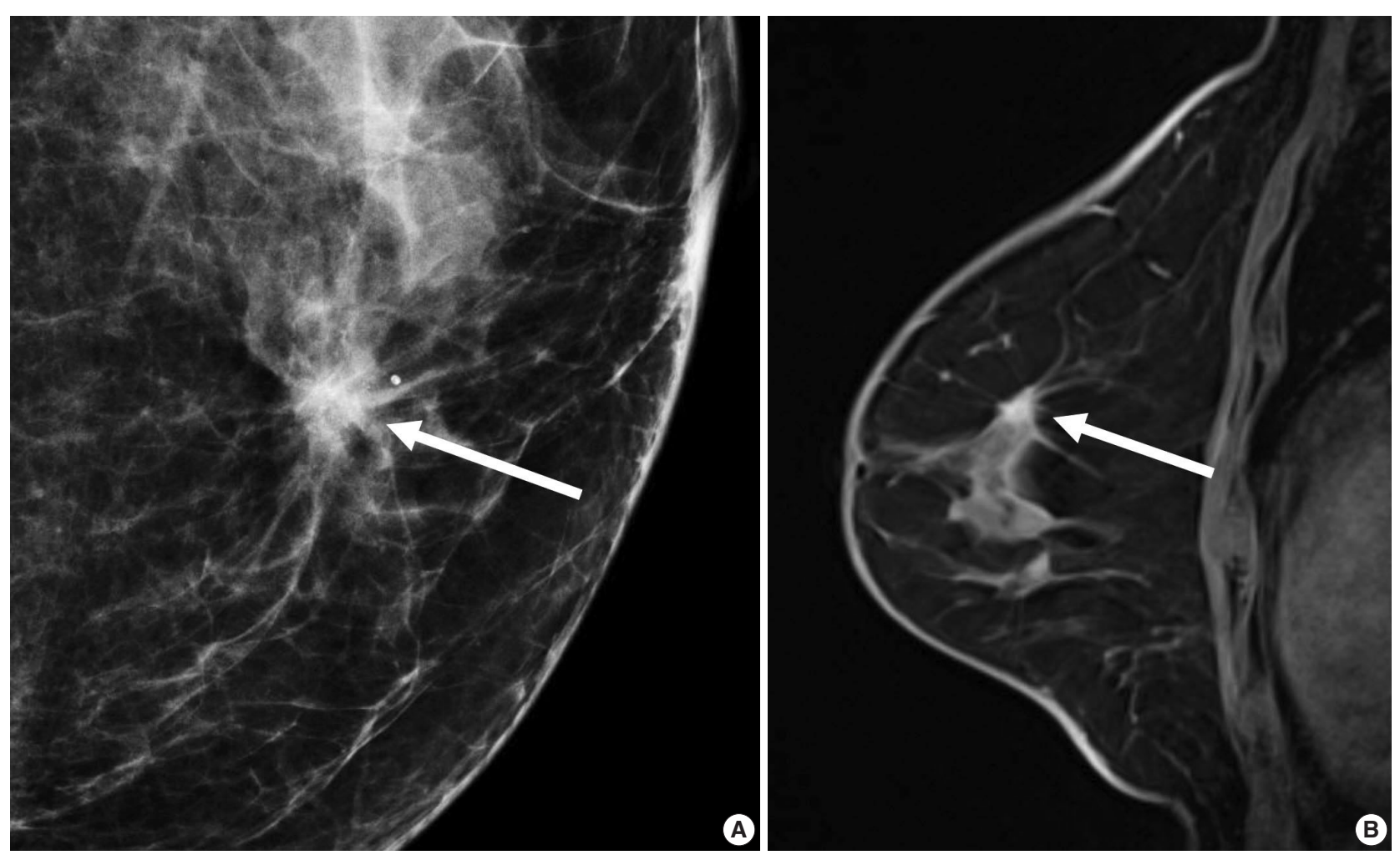

Fig. 1. A 56-year-old woman with a luminal A-like breast cancer. (A) Mammography shows a spiculated mass with calcifications (arrow). (B) Enhanced T1-weighted magnetic resonance imaging shows an irregular, spiculated mass (arrow). Histopathology revealed a 1.5-cm invasive ductal carcinoma with low histologic grade. American Joint Committee on Cancer (AJCC) anatomic stage was T1N0M0. Immunohistochemistry analysis revealed that estrogen receptor $90 \%$ positive, progesterone receptor $1 \%$ positive, human epidermal growth factor receptor $2-$ negative, and Ki-67, 1\% positive. Multigene assay recurrence score was 10 and low risk. The 9-year distant recurrence risk was estimated as 3\%. She did not receive adjuvant chemotherapy, but received aromatase inhibitor. 
low us to distinguish prognosis of patients according to the proliferation-associated genes expression levels [24]. However, the signatures do not apply to patients with ER-negative tumors, because more than $95 \%$ of them already have high expression of proliferation-associated genes [24,25]. The oncotype DX assay analyzes a panel of 21 genes to decide a recurrence score (RS) representing the possibility of recurrent cancer within 10 years. Adjuvant chemotherapy provides greater benefits for patients with high-RS tumors and does very little for patients with lowRS tumors (Fig. 1) [26].

In morphologic analyses for mammography, ER-positive tumor tends to show a not-circumscribed margin (Fig. 1), which is in contrast to ER-negative tumors $[12,13,27]$. Tumor roundness score, quantifying the relative similarity to a perfect circle, has been shown to have an inverse correlation with the ER expression (\%) and a positive correlation with the Ki-67 index [14]. By ultrasonography, parallel orientation (odds ratio [OR], 5.53; $\mathrm{p}=.02)$ and tumor roundness (OR, 1.70 per 10 increase in the roundness value; $\mathrm{p}=.01$ ) were independent features associated with high RS on Oncotype DX [28]. The high-risk group was also associated with the presence of calcifications, similar to a previous study in which a mass with pleomorphic microcalcifications might be associated with an intermediate to high RS in ER-positive, HER2-negative early breast cancer at mammography [29].

A previous report on MRI results demonstrated that patients with luminal B subtype tended to have multifocal or multicentric cancer 2.8 times more often than patients with luminal A subtype [30]. Sutton et al. [15] reported that an increased kurtosis was associated with high RS on Oncotype DX for ER-positive/ HER2-negative tumors (Fig. 2). Kurtosis is a second order parameter quantifying the amount of histogram deviating from a Gaussian shape. High kurtosis might reflect the amount of heterogeneity in a complex way, and these tumors are believed to be more biologically aggressive [19]. This result is in line with a study reporting that both ER-positive and ER-negative tumors showed statistically different entropy levels [16]. The entropy reflecting spatial distribution pattern of grayness of voxel is also believed to be an important biomarker at textural analysis of medical image.
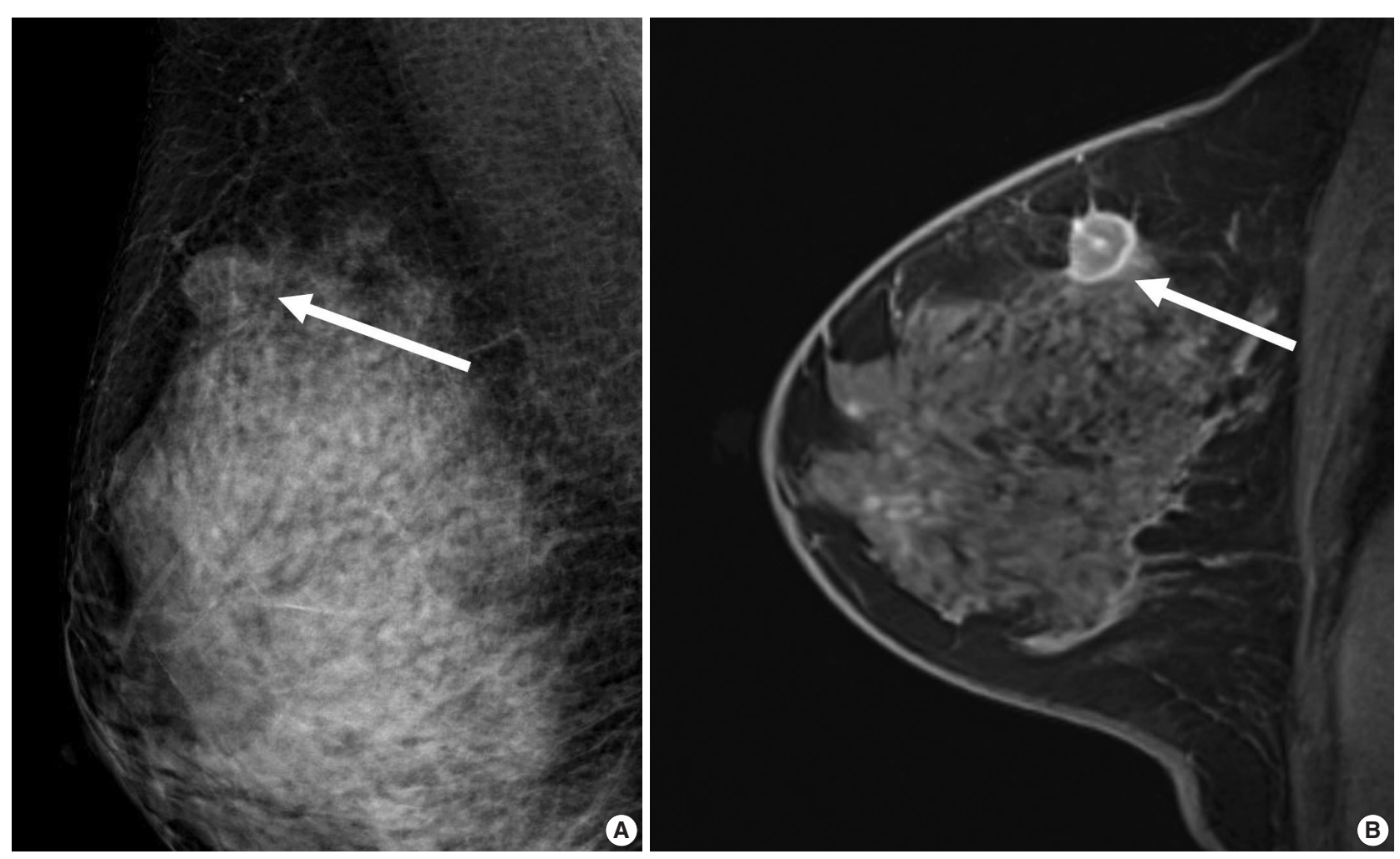

Fig. 2. A 44-year-old woman with a luminal A-like breast cancer. (A) Mammography shows an oval non-calcified mass (arrow). (B) Enhanced T1-weighted magnetic resonance imaging shows an irregular mass with internal rim-enhancement (arrow). Histopathology revealed a 1.7-cm invasive ductal carcinoma with intermediate histologic grade. American Joint Committee on Cancer (AJCC) anatomic stage was T1N0M0. Immunohistochemistry analysis revealed that estrogen receptor $90 \%$ positive, progesterone receptor $5 \%$ positive, human epidermal growth factor receptor 2 negative, and Ki-67, 4\% positive. Multigene assay recurrence score was 23. The 10-year distant recurrence risk was estimated as $12 \%$ and high risk. She received adjuvant chemotherapy and tamoxifen. 

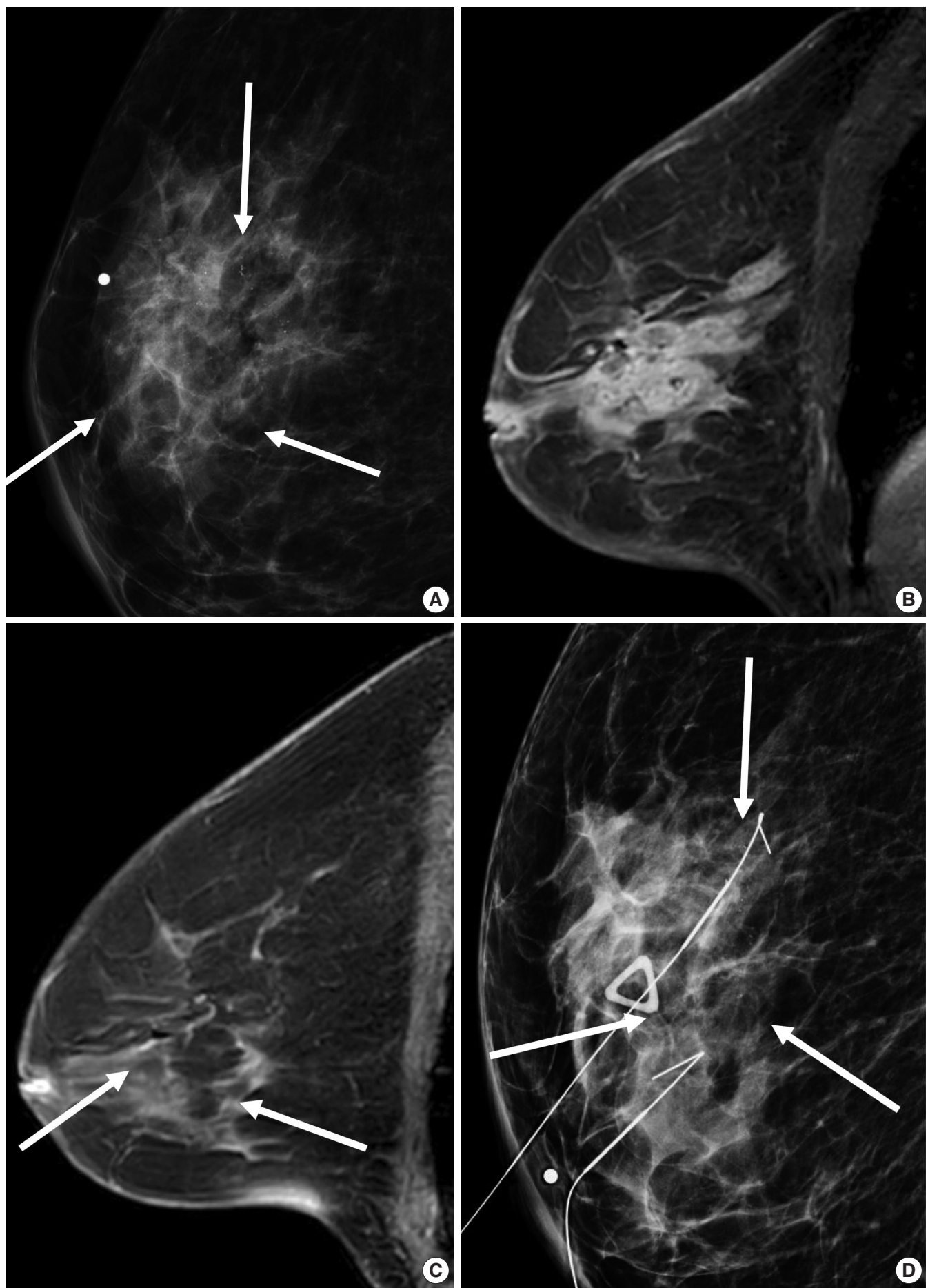

Fig. 3. A 67-year-old woman with a human epidermal growth factor receptor 2 (HER2)-positive breast cancer. (A) Mammography shows illdefined asymmetry with pleomorphic microcalcifications (arrows). (B) Enhanced T1-weighted magnetic resonance imaging (MRI) shows an 8.2-cm ill-defined, diffuse irregular mass with internal heterogeneous enhancement. Needle biopsy revealed an invasive ductal carcinoma with high histologic grade. Immunohistochemistry analysis revealed that estrogen receptor and progesterone receptor negative, and HER2 positive. (C) Following combined docetaxel, carboplatin and dual HER2 blockade, there is no residual mass and but subtle enhancements in the breast on MRI (arrows). (D) Mammography shows two hookwires around the residual calcifications (arrows). Surgical histopathology revealed pathological complete response in the breast and axilla. 


\section{HUMAN EPIDERMAL GROWTH FACTOR RECEPTOR 2-ENRICHED SUBTYPE}

HER 2 overexpression is found in approximately $20 \%$ of invasive breast cancers. It is associated with worse prognosis but good response to HER2-targeted therapies [31] and is reported to increase cell proliferation, survival, mobility, and invasiveness, as well as neo-angiogenesis at the cellular level [32]. The Cancer Genome Atlas (TCGA) and clinical trials have suggested that HER2-positive tumors are a heterogeneous group of cancers [3]. Compared with ER-positive/HER2-positive tumors, patients with ER-negative/HER2-positive tumors show a higher risk of death within 5 years of diagnosis; the first recurrence in brain was higher and in bone was lower, and the response rate to neoadjuvant chemotherapy was higher [33,34]. HER2-targeted agents combined with chemotherapy are recommended for ER-negative/HER2-positive tumors, and HER2-targeted agents with endocrine therapy are recommended for ER-positive/HER2positive tumors [4]. A higher $\mathrm{pCR}$ rate was observed in patients with ER-negative/HER2-positive tumors than in patients with ER-positive/HER2-positive tumors [35]. The pCR rate was over $70 \%$ using the dual HER2 blockade, either with trastuzumab with lapatinib or trastuzumab with pertuzumab in combination with chemotherapy [6].

HER2 overexpression was also associated with the presence of calcifications, branching or fine linear shape calcifications, high level of suspicion on mammography, and a washout or fast early enhancement kinetic curve pattern on MRI (Fig. 3) [36]. MRI showed that patients with the HER2 subtype tended to have 4.1 times more multifocal or multicentric cancers than patients with the luminal A subtype [30]. Fine pleomorphic/fine linear or linear branching calcification morphology on mammography (OR, 7.23), PR negativity (OR, 6.76), and a high TILs (tumor infiltrating lymphocytes) level (OR, 5.92) were independent factors associated with $\mathrm{pCR}$ in patients receiving neoadjuvant chemotherapy with dual HER2 blockade (Fig. 3) [37]. Low tumor peak enhancement at MRI indicating less aggressiveness was significantly associated with high TILs (OR, 1.01; $\mathrm{p}=.020)$ [38]. These results are consistent with previous studies showing that TILs observed in breast cancers were associated with higher rate of $\mathrm{pCR}$ or improved overall survival outcomes $[5,6]$. Moreover, increasing TILs during systemic therapy was reported to be correlated with pCR [39], which suggests that MRI could provide valuable information regarding response during treatment.

\section{BASAL-LIKE SUBTYPES}

TNBC comprises $10 \%-20 \%$ of all breast cancers. The term of TNBC and basal-like tumors are interchangeably used because $86 \%$ of TNBC are the basal-like subtype [3]. However, each of the intrinsic subtypes exist within a TNBC [40], and TNBC is a very heterogeneous group of tumors based on ge-
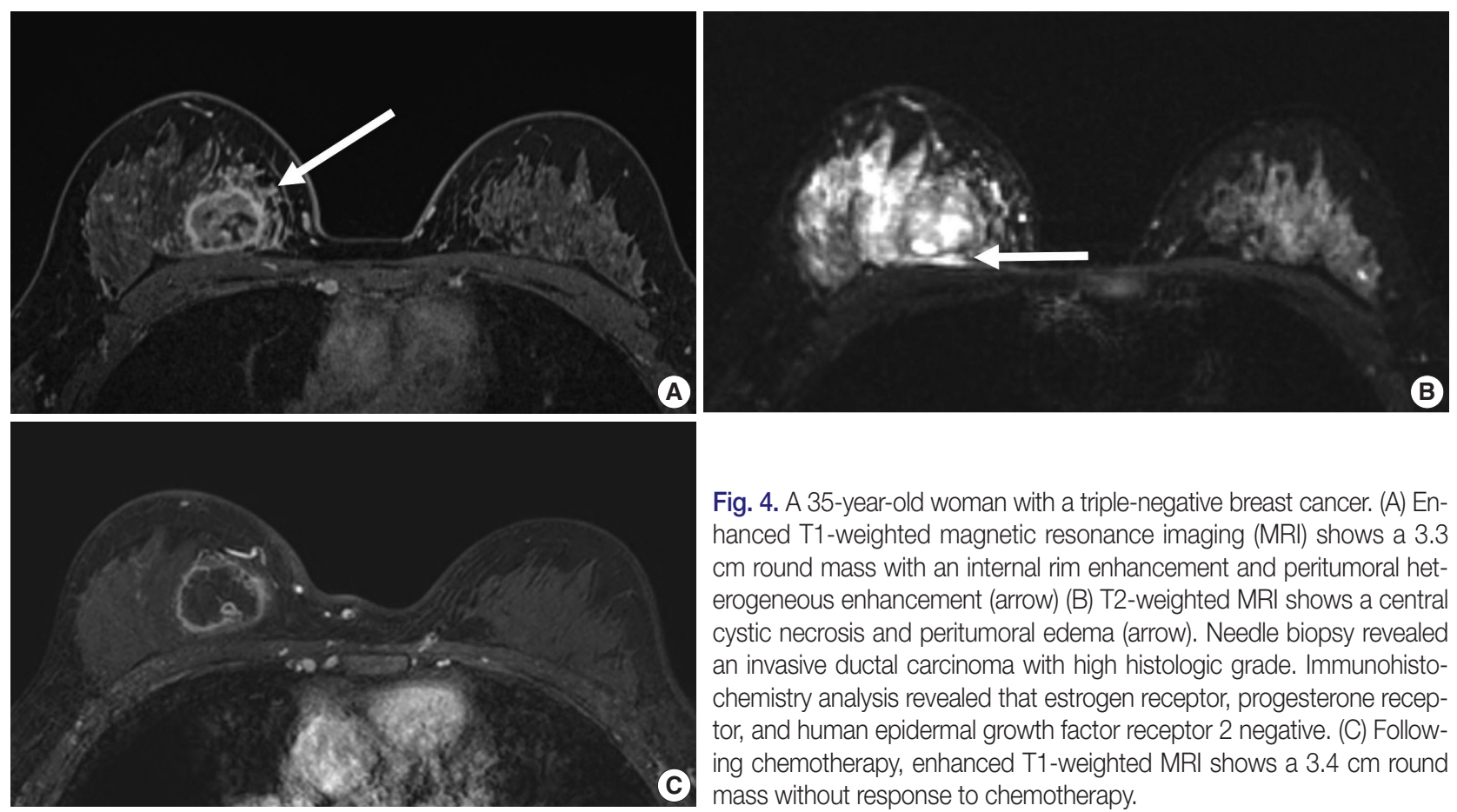

Fig. 4. A 35-year-old woman with a triple-negative breast cancer. (A) Enhanced T1-weighted magnetic resonance imaging (MRI) shows a 3.3 $\mathrm{cm}$ round mass with an internal rim enhancement and peritumoral heterogeneous enhancement (arrow) (B) T2-weighted MRI shows a central cystic necrosis and peritumoral edema (arrow). Needle biopsy revealed an invasive ductal carcinoma with high histologic grade. Immunohistochemistry analysis revealed that estrogen receptor, progesterone receptor, and human epidermal growth factor receptor 2 negative. (C) Following chemotherapy, enhanced T1-weighted MRI shows a $3.4 \mathrm{~cm}$ round mass without response to chemotherapy. 
netic profiling [41]. Rates of $\mathrm{pCR}$ after receiving anthracycline/ taxane regimen are 25\%-35\% and patients with pCR show a better outcome in patients with TNBC [25]. Recent St. Gallen Consensus Conference Guidelines recommended that TILs should be routinely characterized for TNBC in view of their prognostic value [6]. Tumor programmed death-ligand 1 and immune-cell programmed death- 1 expression are considered as markers to predict benefit from immunotherapy for advanced TNBC [6]. Also, in TNBC with residual disease following neoadjuvant chemotherapy, post-neoadjuvant treatment with capecitabine showed survival benefits [42].

Unifocal, circumscribed margin, round shape, and no associated calcifications are signatures of TNBC [43]. Circumscribed margin and round shape are more commonly found in highgrade tumors and spiculation is more frequently found in lowgrade tumors [44]. TNBC has been shown to have a higher tumor roundness score compared with the other subtypes, reflecting a more biologically aggressive tumor type. Absence of calcifications is also considered representative of rapid malignant transformation of TNBC with bypassing of the in-situ stage [43]. TNBC shows a round mass with rim enhancement on MRI and frequently has internal high signal intensity on T2-weighted MRI [45-47] (Fig. 4). Recent studies using radiomics analysis have reported that TNBC masses tend to be larger, have a more heterogeneous enhancement texture, are more irregularly shaped, and have a rapid enhancement rate compared with other subtypes $[17,18]$. Notably, the heterogeneous enhancement texture, quantified at the first post-contrast enhanced MRI, has emerged as a discriminatory indicator for tumor subtype, regardless of tumor size [17,18]. Waugh et al. [16] also reported that TNBC and HER 2 subtypes showed increased entropy values compared with luminal A and luminal B subtypes.

In the neoadjuvant chemotherapy setting, intratumoral necrosis was associated with non-response to chemotherapy [48] and peritumoral edema was associated with worse recurrence-freesurvival outcome of TNBC (Fig. 4) [49]. With textural analysis, increased kurtosis of non-TNBC on T2 weighted image was independently associated with $\mathrm{pCR}$; however, the association between increased kurtosis and $\mathrm{pCR}$ was not found in TNBC [19]. In another study predicting pCR by using radiomics, Braman et al. reported that combined intratumoral and peritumoral radiomic features yielded a maximum AUC of 0.83 for the ERpositive/HER2-negative group and 0.93 for TNBC or HER2positive group [20]. In Braman et al's study [20], elevated peritumoral heterogeneity was associated with non-pCR in ERpositive/HER2-negative tumors, and peritumoral speckled en- hancement pattern was associated with non-pCR in TNBC or HER2-positive tumors (Fig. 4A) [20]. These results are in line with peritumoral lymphatics or vascular invasion, and peritumoral immune response as predictors of survival. In addition, TILs, known as a favorable prognostic factor in TNBC, could be quantified by textural analysis [20]. Thus, peritumoral radiomics features on MRI could be valuable predictors of $\mathrm{pCR}$ in TNBC and HER2-positive tumors.

\section{CONCLUSION}

Breast cancer consists of heterogeneous subtypes and evolves continuously after systemic therapy. Earlier studies linking imaging features and molecular subtypes have reported presence of calcifications, margin or shape features, and enhancement features on dynamic contrast enhanced MRI according to each subtype. Recent studies using radiomics parameters, which are indiscernible by the human eye, have shown high accuracy in distinguishing molecular subtypes, prediction of responses to chemotherapy, and prediction of survival outcomes. Imaging biomarkers could be helpful in realizing better precision medicine due to the feasibility of repeated measurements for whole tumors and the applicability of deep-learning based algorithms.

\section{Ethics Statement}

Not applicable.

\section{ORCID}

Nariya Cho https://orcid.org/0000-0003-4290-2777

\section{Conflicts of Interest}

The authors declare that they have no potential conflicts of interest.

\section{Funding Statement}

No funding to declare.

\section{References}

1. Cancer Genome Atlas Network. Comprehensive molecular portraits of human breast tumours. Nature 2012; 490: 61-70.

2. Sorlie T, Perou CM, Tibshirani R, et al. Gene expression patterns of breast carcinomas distinguish tumor subclasses with clinical implications. Proc Natl Acad Sci U S A 2001; 98: 10869-74.

3. Prat A, Pineda E, Adamo B, et al. Clinical implications of the intrinsic molecular subtypes of breast cancer. Breast 2015; 24 Suppl 2: S26-35.

4. Coates AS, Winer EP, Goldhirsch A, et al. Tailoring therapies--improving the management of early breast cancer: St Gallen International Expert Consensus on the Primary Therapy of Early Breast Cancer 2015. Ann Oncol 2015; 26: 1533-46.

5. Curigliano G, Burstein HJ, Winer EP, et al. De-escalating and escalating treatments for early-stage breast cancer: the St. Gallen Inter- 
national Expert Consensus Conference on the Primary Therapy of Early Breast Cancer 2017. Ann Oncol 2017; 28: 1700-12.

6. Burstein HJ, Curigliano G, Loibl S, et al. Estimating the benefits of therapy for early-stage breast cancer: the St. Gallen International Consensus Guidelines for the primary therapy of early breast cancer 2019. Ann Oncol 2019; 30: 1541-57.

7. Cho N. Molecular subtypes and imaging phenotypes of breast cancer. Ultrasonography 2016; 35: 281-8.

8. Lee SH, Park H, Ko ES. Radiomics in breast imaging from techniques to clinical applications: a review. Korean J Radiol 2020; 21: 779-92.

9. Leithner D, Horvat JV, Marino MA, et al. Radiomic signatures with contrast-enhanced magnetic resonance imaging for the assessment of breast cancer receptor status and molecular subtypes: initial results. Breast Cancer Res 2019; 21: 106.

10. Kim S, Kim MJ, Kim EK, Yoon JH, Park VY. MRI radiomic features: association with disease-free survival in patients with triplenegative breast cancer. Sci Rep 2020; 10: 3750.

11. Song L, Lu H, Yin J. Preliminary study on discriminating HER2 2+ amplification status of breast cancers based on texture features semi-automatically derived from pre-, post-contrast, and subtraction images of DCE-MRI. PLoS One 2020; 15: e0234800.

12. Mazurowski MA, Zhang J, Grimm LJ, Yoon SC, Silber JI. Radiogenomic analysis of breast cancer: luminal B molecular subtype is associated with enhancement dynamics at MR imaging. Radiology 2014; 273: 365-72.

13. Grimm LJ, Zhang J, Mazurowski MA. Computational approach to radiogenomics of breast cancer: Luminal A and luminal B molecular subtypes are associated with imaging features on routine breast MRI extracted using computer vision algorithms. J Magn Reson Imaging 2015; 42: 902-7.

14. Bae MS, Seo M, Kim KG, Park IA, Moon WK. Quantitative MRI morphology of invasive breast cancer: correlation with immunohistochemical biomarkers and subtypes. Acta Radiol 2015; 56: 269-75.

15. Sutton EJ, Oh JH, Dashevsky BZ, et al. Breast cancer subtype intertumor heterogeneity: MRI-based features predict results of a genomic assay. J Magn Reson Imaging 2015; 42: 1398-406.

16. Waugh SA, Purdie CA, Jordan LB, et al. Magnetic resonance imaging texture analysis classification of primary breast cancer. Eur Radiol 2016; 26: 322-30.

17. Li H, Zhu Y, Burnside ES, et al. Quantitative MRI radiomics in the prediction of molecular classifications of breast cancer subtypes in the TCGA/TCIA data set. NPJ Breast Cancer 2016; 2: 16012.

18. Agner SC, Rosen MA, Englander S, et al. Computerized image analysis for identifying triple-negative breast cancers and differentiating them from other molecular subtypes of breast cancer on dynamic contrast-enhanced MR images: a feasibility study. Radiology 2014; 272: 91-9.

19. Chamming's F, Ueno Y, Ferre R, et al. Features from computerized texture analysis of breast cancers at pretreatment MR imaging are associated with response to neoadjuvant chemotherapy. Radiology 2018; 286: 412-20.

20. Braman NM, Etesami M, Prasanna P, et al. Intratumoral and peritumoral radiomics for the pretreatment prediction of pathological complete response to neoadjuvant chemotherapy based on breast DCE-MRI. Breast Cancer Res 2017; 19: 57.

21. Chia SK, Bramwell VH, Tu D, et al. A 50-gene intrinsic subtype classifier for prognosis and prediction of benefit from adjuvant tamoxi- fen. Clin Cancer Res 2012; 18: 4465-72.

22. Prat A, Parker JS, Fan C, et al. Concordance among gene expression-based predictors for ER-positive breast cancer treated with adjuvant tamoxifen. Ann Oncol 2012; 23: 2866-73.

23. Iwamoto T, Booser D, Valero V, et al. Estrogen receptor (ER) mRNA and ER-related gene expression in breast cancers that are $1 \%$ to $10 \%$ ER-positive by immunohistochemistry. J Clin Oncol 2012; 30: 72934.

24. Ng CK, Schultheis AM, Bidard FC, Weigelt B, Reis-Filho JS. Breast cancer genomics from microarrays to massively parallel sequencing: paradigms and new insights. J Natl Cancer Inst 2015; 107:djv015.

25. Liedtke C, Mazouni C, Hess KR, et al. Response to neoadjuvant therapy and long-term survival in patients with triple-negative breast cancer. J Clin Oncol 2008; 26: 1275-81.

26. Paik S, Tang G, Shak S, et al. Gene expression and benefit of chemotherapy in women with node-negative, estrogen receptor-positive breast cancer. J Clin Oncol 2006; 24: 3726-34.

27. Shin HJ, Kim HH, Huh MO, et al. Correlation between mammographic and sonographic findings and prognostic factors in patients with node-negative invasive breast cancer. Br J Radiol 2011; 84: 19-30.

28. Chae EY, Moon WK, Kim HH, et al. Association between ultrasound features and the 21-gene recurrence score cssays in patients with oestrogen receptor-positive, HER2-negative, invasive breast cancer. PLoS One 2016; 11: e0158461.

29. Yepes MM, Romilly AP, Collado-Mesa F, et al. Can mammographic and sonographic imaging features predict the Oncotype DX recurrence score in $\mathrm{T} 1$ and $\mathrm{T} 2$, hormone receptor positive, HER 2 negative and axillary lymph node negative breast cancers? Breast Cancer Res Treat 2014; 148: 117-23.

30. Grimm LJ, Johnson KS, Marcom PK, Baker JA, Soo MS. Can breast cancer molecular subtype help to select patients for preoperative MR imaging? Radiology 2015; 274: 352-8.

31. Arteaga CL, Sliwkowski MX, Osborne CK, Perez EA, Puglisi F, Gianni L. Treatment of HER2-positive breast cancer: current status and future perspectives. Nat Rev Clin Oncol 2011; 9: 16-32.

32. Zhou BP, Hung MC. Dysregulation of cellular signaling by HER2/ neu in breast cancer. Semin Oncol 2003; 30: 38-48.

33. Perez EA, Romond EH, Suman VJ, et al. Four-year follow-up of trastuzumab plus adjuvant chemotherapy for operable human epidermal growth factor receptor 2-positive breast cancer: joint analysis of data from NCCTG N9831 and NSABP B-31. J Clin Oncol 2011; 29: 3366-73.

34. Vaz-Luis I, Ottesen RA, Hughes ME, et al. Impact of hormone receptor status on patterns of recurrence and clinical outcomes among patients with human epidermal growth factor-2-positive breast cancer in the National Comprehensive Cancer Network: a prospective cohort study. Breast Cancer Res 2012; 14: R129.

35. Gianni L, Eiermann W, Semiglazov V, et al. Neoadjuvant and adjuvant trastuzumab in patients with HER2-positive locally advanced breast cancer (NOAH): follow-up of a randomised controlled superiority trial with a parallel HER2-negative cohort. Lancet Oncol 2014; 15: 640-7.

36. Elias SG, Adams A, Wisner DJ, et al. Imaging features of HER2 overexpression in breast cancer: a systematic review and metaanalysis. Cancer Epidemiol Biomarkers Prev 2014; 23: 1464-83.

37. Yoon GY, Chae EY, Cha JH, et al. Imaging and clinicopathologic features associated with pathologic complete response in HER2positive breast cancer receiving neoadjuvant chemotherapy with 
dual HER2 blockade. Clin Breast Cancer 2020; 20: 25-32.

38. Choi WJ, Kim Y, Cha JH, et al. Correlation between magnetic resonance imaging and the level of tumor-infiltrating lymphocytes in patients with estrogen receptor-negative HER2-positive breast cancer. Acta Radiol 2020; 61: 3-10.

39. Nuciforo P, Pascual T, Cortes J, et al. A predictive model of pathologic response based on tumor cellularity and tumor-infiltrating lymphocytes (CelTIL) in HER2-positive breast cancer treated with chemo-free dual HER2 blockade. Ann Oncol 2018; 29: 170-7.

40. Prat A, Adamo B, Cheang MC, Anders CK, Carey LA, Perou CM. Molecular characterization of basal-like and non-basal-like triplenegative breast cancer. Oncologist 2013; 18: 123-33.

41. Lehmann BD, Bauer JA, Chen X, et al. Identification of human triple-negative breast cancer subtypes and preclinical models for selection of targeted therapies. J Clin Invest 2011; 121: 2750-67.

42. Masuda N, Lee SJ, Ohtani S, et al. Adjuvant capecitabine for breast cancer after preoperative chemotherapy. N Engl J Med 2017; 376: 2147-59.

43. Dogan BE, Turnbull LW. Imaging of triple-negative breast cancer. Ann Oncol 2012; 23 Suppl 6: vi23-9.

44. Lamb PM, Perry NM, Vinnicombe SJ, Wells CA. Correlation between ultrasound characteristics, mammographic findings and his- tological grade in patients with invasive ductal carcinoma of the breast. Clin Radiol 2000; 55: 40-4.

45. Uematsu T, Kasami M, Yuen S. Triple-negative breast cancer: correlation between MR imaging and pathologic findings. Radiology 2009; 250: 638-47.

46. Youk JH, Son EJ, Chung J, Kim JA, Kim EK. Triple-negative invasive breast cancer on dynamic contrast-enhanced and diffusionweighted MR imaging: comparison with other breast cancer subtypes. Eur Radiol 2012; 22: 1724-34.

47. Kim GR, Ku YJ, Cho SG, Kim SJ, Min BS. Associations between gene expression profiles of invasive breast cancer and Breast Imaging Reporting and Data System MRI lexicon. Ann Surg Treat Res 2017; 93: 18-26.

48. Kawashima H, Inokuchi M, Furukawa H, Kitamura S. Triple-negative breast cancer: are the imaging findings different between responders and nonresponders to neoadjuvant chemotherapy? Acad Radiol 2011; 18: 963-9.

49. Bae MS, Shin SU, Ryu HS, et al. Pretreatment MR imaging features of triple-negative breast cancer: association with response to neoadjuvant chemotherapy and recurrence-free survival. Radiology 2016; 281: $392-400$. 